\title{
Direct Chemical Reduction of Neptunium Oxide to Neptunium Metal using Calcium and Calcium Chloride
}

\author{
Leah N. Squires ${ }^{\mathrm{a}}$, Paul Lessing ${ }^{\mathrm{a}}$
}

a. Idaho National Laboratory 2525 Fremont Avenue, Idaho Falls, ID 83415

\begin{abstract}
A process of direct reduction of neptunium oxide to neptunium metal using calcium metal as the reducing agent is discussed. After reduction of the oxide to metal, the metal is separated by density from the other components of the reaction mixture and can be easily removed upon cooling. The direct reduction technique consistently produces high purity ( $98 \%-99 \%$ pure) neptunium metal.
\end{abstract}




\section{Contents}

Abstract

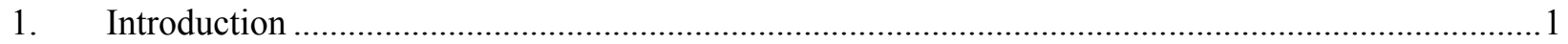

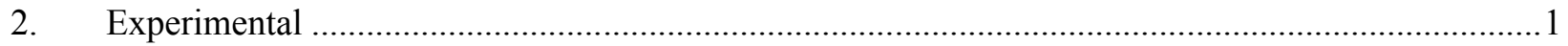

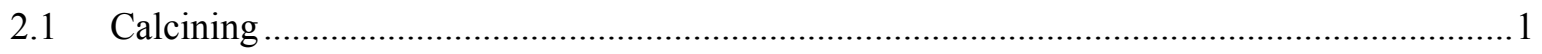

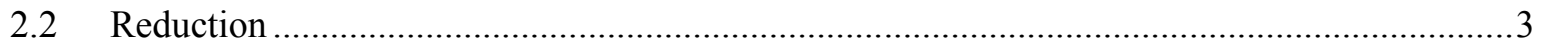

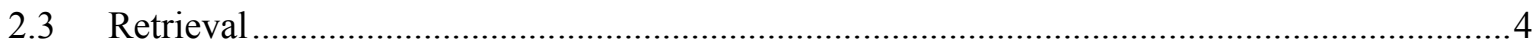

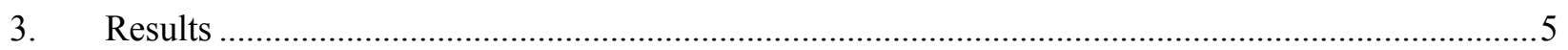

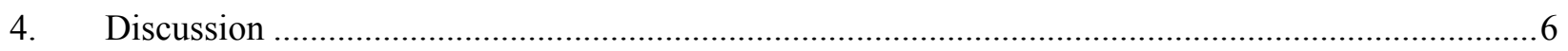

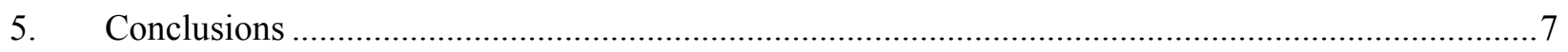

\section{Figures}

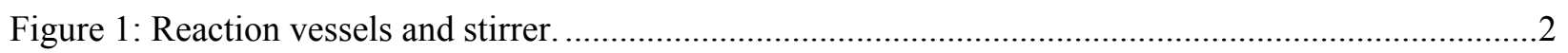

Figure 2. The hot uniaxial press (HUP) furnace insert apparatus.......................................................... 3

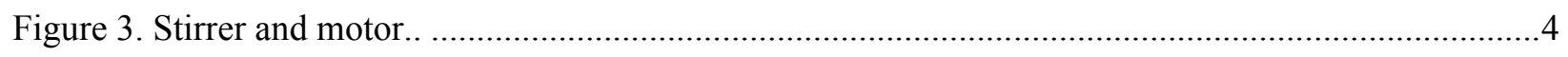

Figure 4. Reaction mixture with crucible removed. ......................................................................6

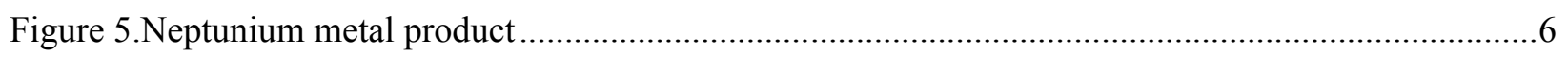

\section{Tables}

Table 1. Densities of the materials in the reaction mixture. 


\section{Introduction}

A key to reducing the long term health and safety hazards presented by the storage of spent nuclear fuel is reduction or elimination of the minor actinides in the fuel such as neptunium, americium and curium. One method currently under investigation for the elimination of these elements is transmutation; whereby, the minor actinides are incorporated into new fuel which is placed in a fast reactor where the minor actinides of interest fission to shorter lived products. In order to develop the process of transmutation it is necessary to first fabricate small quantities of fuel with minor actinide additives and perform thorough characterization and irradiation testing on it.

Neptunium metal is a key component in the fabrication of transmutation fuels and, currently, there is very little neptunium metal available. The majority of the neptunium that is available is in the oxide form and much of it is of questionable purity. It is imperative to the future of transmutation development that high purity neptunium metal is obtained to be incorporated as an additive to approximate spent fuel. In order to produce the needed material from that which is available, a relatively high throughput process of reducing neptunium oxide to neptunium metal was developed. A number of literature references describe methods of reducing plutonium or uranium oxide to metal using calcium $[1,2,3,4]$ or lithium $[3,5,6]$ as the reducing agent. A process of reducing americium oxide to americium metal using lithium [7] was also developed; however, no previous work has applied these processes to neptunium. Thus, a method was developed to directly reduce neptunium oxide to neptunium metal using calcium as a reducing agent. A number of experiments were performed to optimize the process. In this process the reagents are heated, stirred and separated by density. This density separation results in a relatively easily retrievable button of neptunium metal that settles to the bottom of the mixture. This process consistently produces high purity (98\%-99\%) neptunium metal.

\section{Experimental}

This work is performed using the Hot Uniaxial Press (HUP) furnace in the Casting Laboratory glovebox of the Analytical Laboratory at the Materials and Fuels Complex of Idaho National Laboratory (INL). The HUP is a water cooled well furnace that extends below the glovebox floor and is loaded from the top. The glovebox is kept under an argon atmosphere. Oxygen levels are monitored constantly within the glovebox and must be below 50ppm in order for this work to be performed. The reagents are placed in a magnesium oxide crucible (Figure 1b) that is custom made to fit inside the furnace insert (crucibles fabricated by Ozark Ceramics, Webb City, MO). Magnesium oxide was chosen as the crucible material because it can withstand the temperatures to which the mixture must be heated for the reduction process, and it does not react with any of the reagents being used in the process. This magnesium oxide crucible is placed inside a stainless steel crucible that is designed to ensure that the contents of the magnesium oxide crucible do not leak into the furnace itself if the magnesium oxide crucible becomes compromised during heating (Figure 1a). The stainless steel crucible is designed to fit inside the HUP furnace stirring insert (Figure 2). The reagents are stirred at temperature using a tantalum stirrer that is fabricated in house at INL. The stirrer is designed with lower impellers to push material up and upper impellers to push material down in order to facilitate mixing of all reagents (Figure 1c).

\subsection{Calcining}

The first step of the process is a calcining heat treatment of the neptunium oxide starting material. In this step, approximately $20 \mathrm{~g}$ of neptunium oxide powder is placed in the magnesium oxide crucible that is then placed in the stainless steel crucible and next placed into the furnace insert. The entire insert is then lowered into the well furnace and heated to $800^{\circ} \mathrm{C}$. The material is held at temperature for six hours. The purpose of the calcining step is to drive off volatile impurities before the reduction. While no specific analysis has been performed on the starting material, it is most likely that a very small amount of water is being driven off during the calcining process. The furnace is turned off after six hours, and the contents are allowed to cool overnight. The following day the material is removed. The neptunium oxide is 
reweighed and the magnesium oxide crucible is examined for damage. If the crucible is not found to be damaged it is used for the reduction process. If any damage is detected, a new crucible is used for the reduction step.

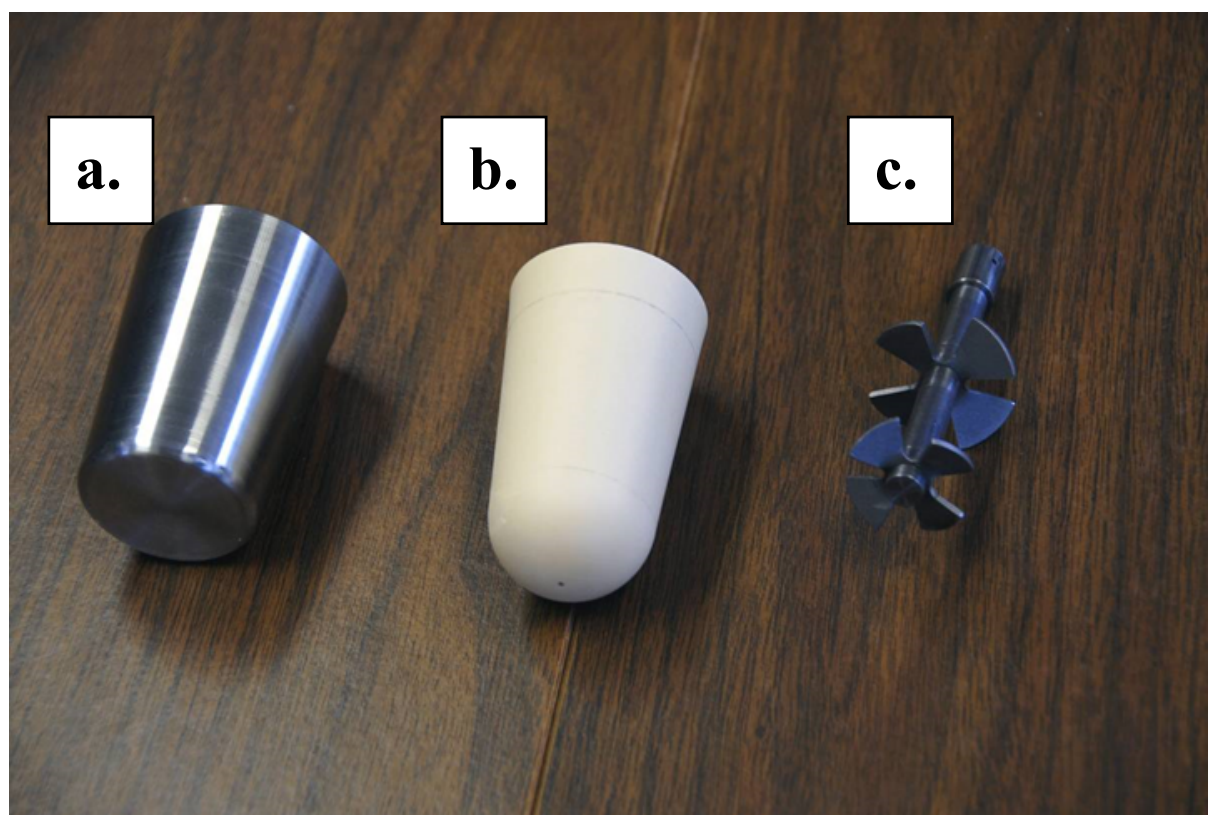

Figure 1: Reaction vessels and stirrer. (a) Stainless steel crucible that fits into the furnace apparatus and protects the furnace well if the magnesium oxide crucible is compromised during heating; (b) heat resistant, non-reactive magnesium oxide crucible that serves as the reaction vessel for the neptunium oxide reduction process; and (c) tantalum stirrer designed with lower impellers to push reagents up and upper impellers to push reagents down in order to promote mixing of the molten material. 


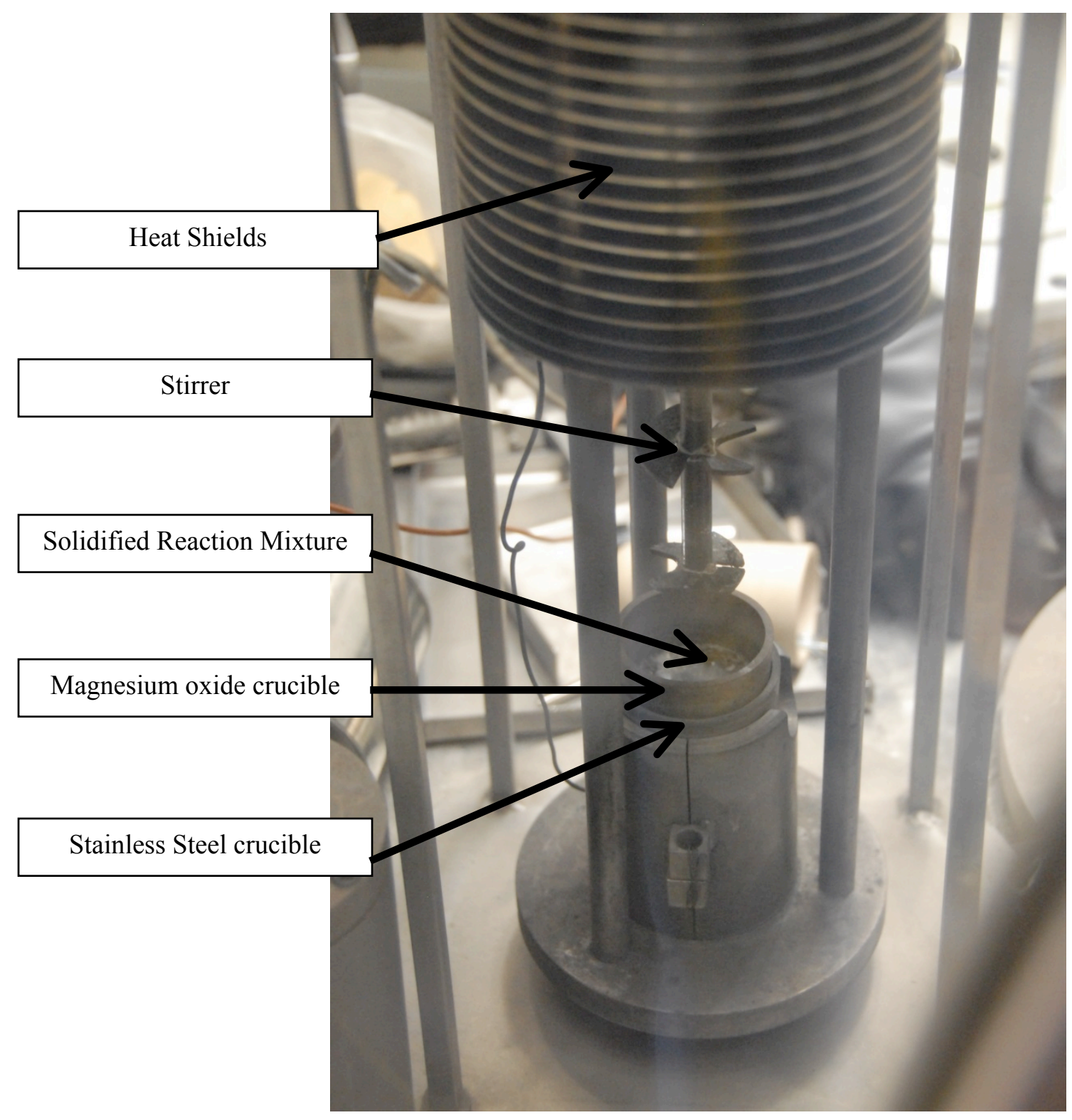

Figure 2. The hot uniaxial press (HUP) furnace insert apparatus. Shown here is a loaded magnesium oxide crucible inside a stainless steel crucible with stirrer suspended above the reaction vessel.

\subsection{Reduction}

The crucible is then refilled for the reduction step of the process. First the tantalum stirrer (Figure 1c) that is designed to ensure thorough mixing of the material is placed in the crucible. It is important to place the stirrer in first because, once the crucible is full of reagents, it is not possible to insert the stirrer. Approximately $9 \mathrm{~g}$ of calcium metal (Fisher Scientific-Alfa Aesar) is placed in the bottom of the crucible, followed by a thin layer of approximately $1 \mathrm{~g}$ of ultra-dry calcium chloride (Fisher Scientific-Alfa Aesar). The calcined neptunium oxide is then placed on top of the thin calcium chloride layer, and the remaining volume of the magnesium oxide crucible is then filled with ultra-dry calcium chloride salt (approximately $100 \mathrm{~g})$.

The furnace insert is placed inside the furnace well and the furnace is heated at a ramp rate of $50^{\circ} \mathrm{C}$ per minute to the maximum heat capacity of the furnace $\left(1000^{\circ} \mathrm{C}\right)$. When the internal temperature 
measured by a thermocouple placed just above the reaction mixture reaches $850^{\circ} \mathrm{C}$, the external gear on the motor is engaged with the gear on the stirrer to begin turning the tantalum stirrer (Figure 3 ). The rate of stirring is $100 \mathrm{RPM}$. The reaction is allowed to sit at temperature for 6 hours with stirring. Once the time has elapsed, the stirrer is removed from the molten mixture before the furnace is shut off to ensure that it does not become lodged in the re-solidified material. After the stirrer is removed the mixture is left at temperature for at least 1 additional hour in order to allow the components of the reaction mixture to settle and separate by density before the furnace is turned off. The crucible and contents are then left in the furnace to cool overnight.

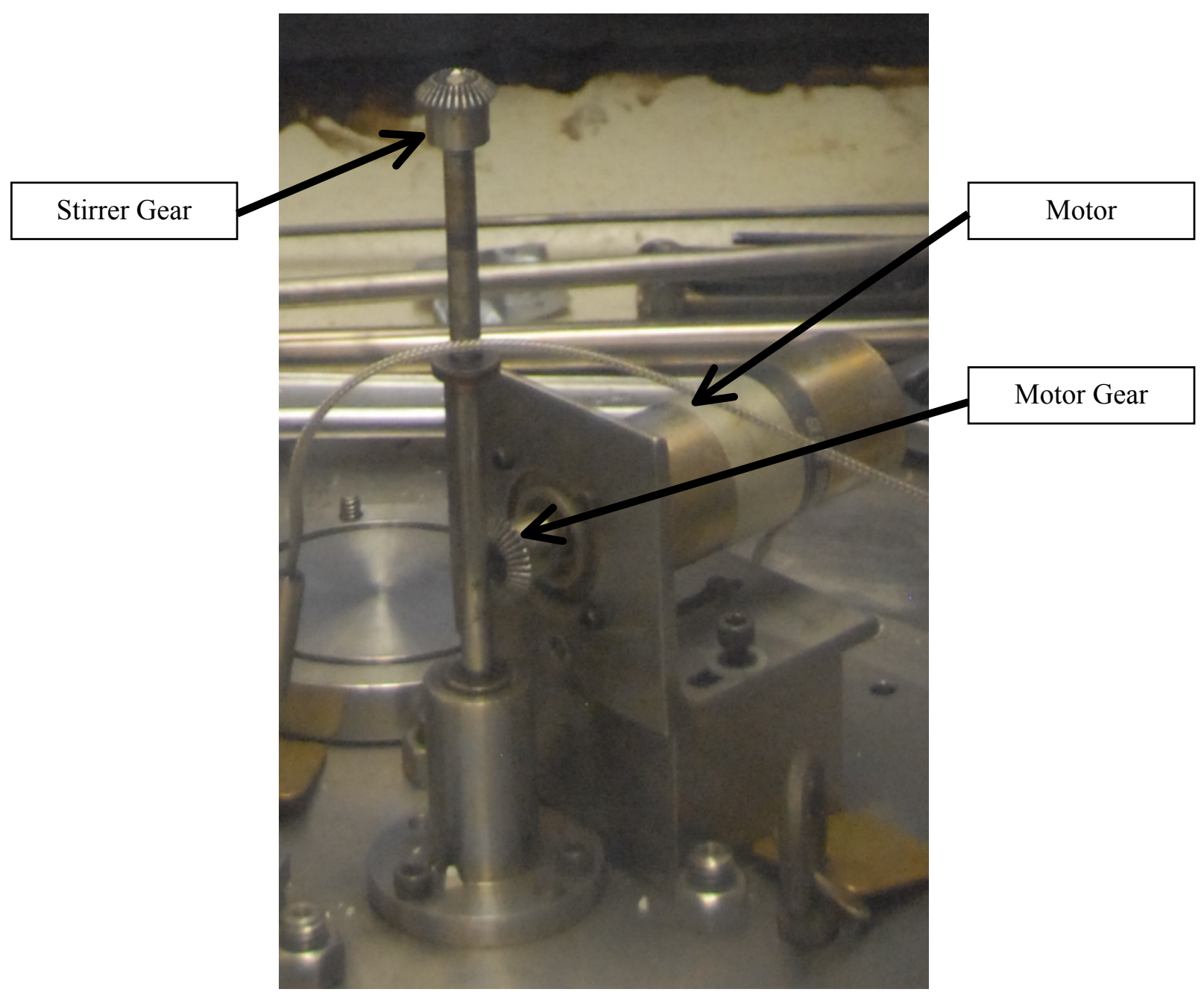

Figure 3. Stirrer and motor. The motor to run the stirrer is located above the furnace well. The stirrer is positioned when reagents are molten and the gear on the stirrer is engaged with the gear on the motor.

\subsection{Retrieval}

Once the reaction mixture is cool (minimum cooling time is overnight), the furnace insert is lifted from the well of the furnace and the magnesium oxide crucible is removed from the furnace insert and the stainless steel crucible. A hammer and chisel are then used to break open the magnesium oxide crucible and remove the material inside. The neptunium metal is present as a small button at the bottom of the reaction mixture. 


\section{Results}

Visual examination of the surface of the reduction mixture once it is cooled and removed from the furnace reveals recrystallized salt material that appears as long, thin crystals on the top of the mixture. Once the magnesium oxide crucible is broken away using a hammer and chisel distinct layers can be seen in the solidified mixture (Figure 4). The layers are formed when the components of the mixture are separated by density during the reduction process. Table 1 shows the densities of the materials in the reaction mixture. The densest material, the neptunium metal, forms an easily removable button on the bottom of the solid (Figure 5). Once removed, a sample of the material from the metal button retrieved from the bottom of the solid mixture is submitted for elemental and isotopic analysis. The results of mass spectrometry analysis consistently show $98 \%-99 \%(+/-3 \%)$ neptunium metal obtained from this reduction process. The most likely impurities present in trace amounts are oxygen and chlorine. It is currently not within the laboratory capability to measure oxygen because all dissolutions of transuranic must be done in an air atmosphere glovebox and the material oxidizes very quickly. Additionally, the dissolutions are performed using hydrochloric acid making it difficult to analyze for chlorine. It is important to note that the error for the analysis technique is $+/-3 \%$ and these samples were determined to be $98 \%-99 \%$ pure so any impurities are present only in trace amounts. The process yield ranges between $50-80 \%$ with an average of $9 \mathrm{~g}$ of metal produced in each run.

Table 1. Published densities of the materials in the reaction mixture

\begin{tabular}{|l|c|}
\hline & Density $\left(\mathrm{g} / \mathrm{cm}^{3}\right)$ \\
\hline Calcium metal & 1.54 \\
\hline Calcium chloride & 2.15 \\
\hline Calcium oxide & 3.35 \\
\hline Neptunium oxide & 11.1 \\
\hline Neptunium metal & 20.45 \\
\hline
\end{tabular}



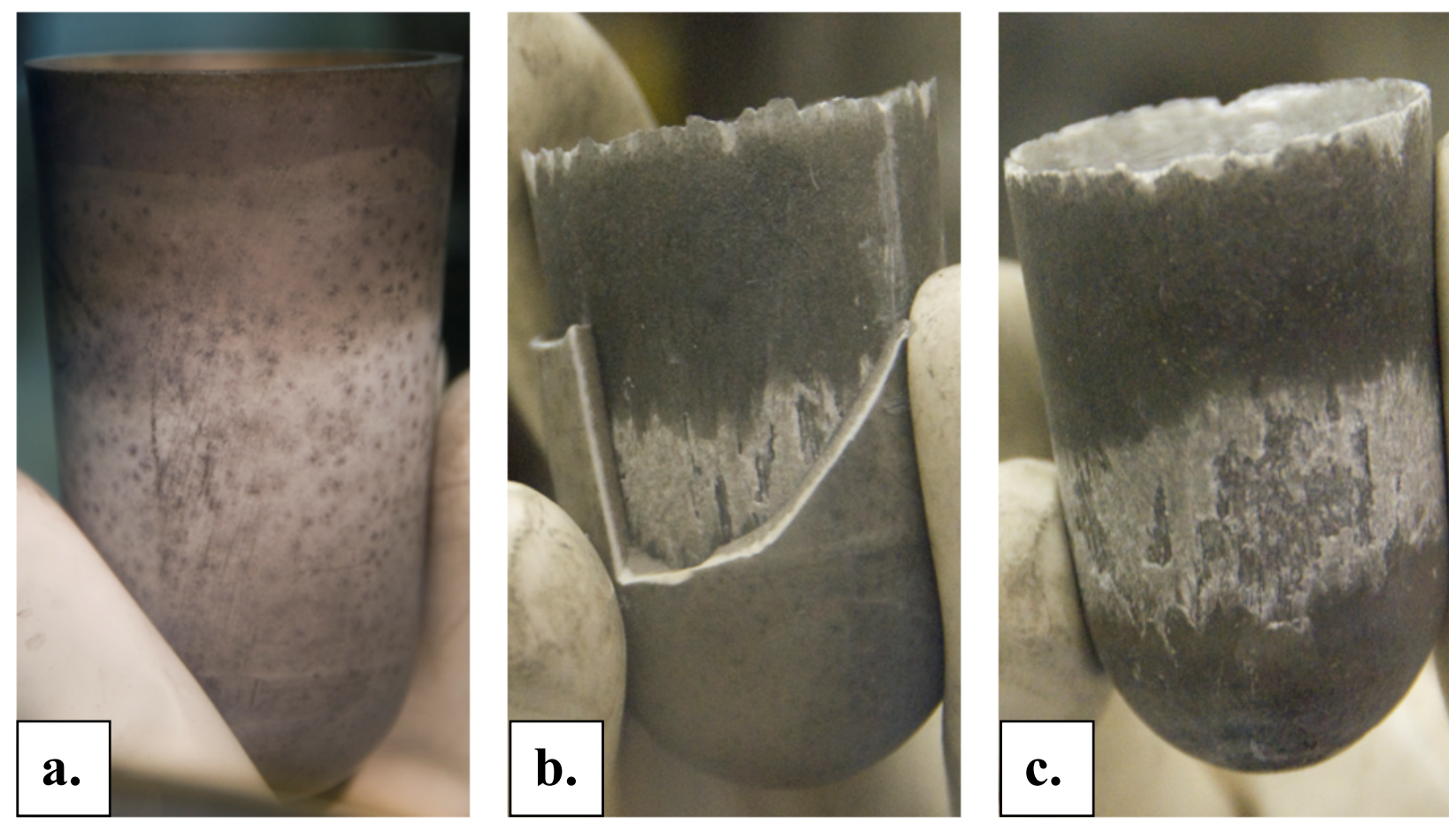

Figure 4. Reaction mixture with crucible removed. (a) The magnesium oxide crucible with contents after it is removed from the stainless steel crucible and furnace insert. (b) The magnesium oxide crucible partially removed from the solidified reaction mixture using a hammer and chisel. (c) The magnesium oxide crucible completely removed from the mixture shows layers that are formed by the separation of the reaction components by density.
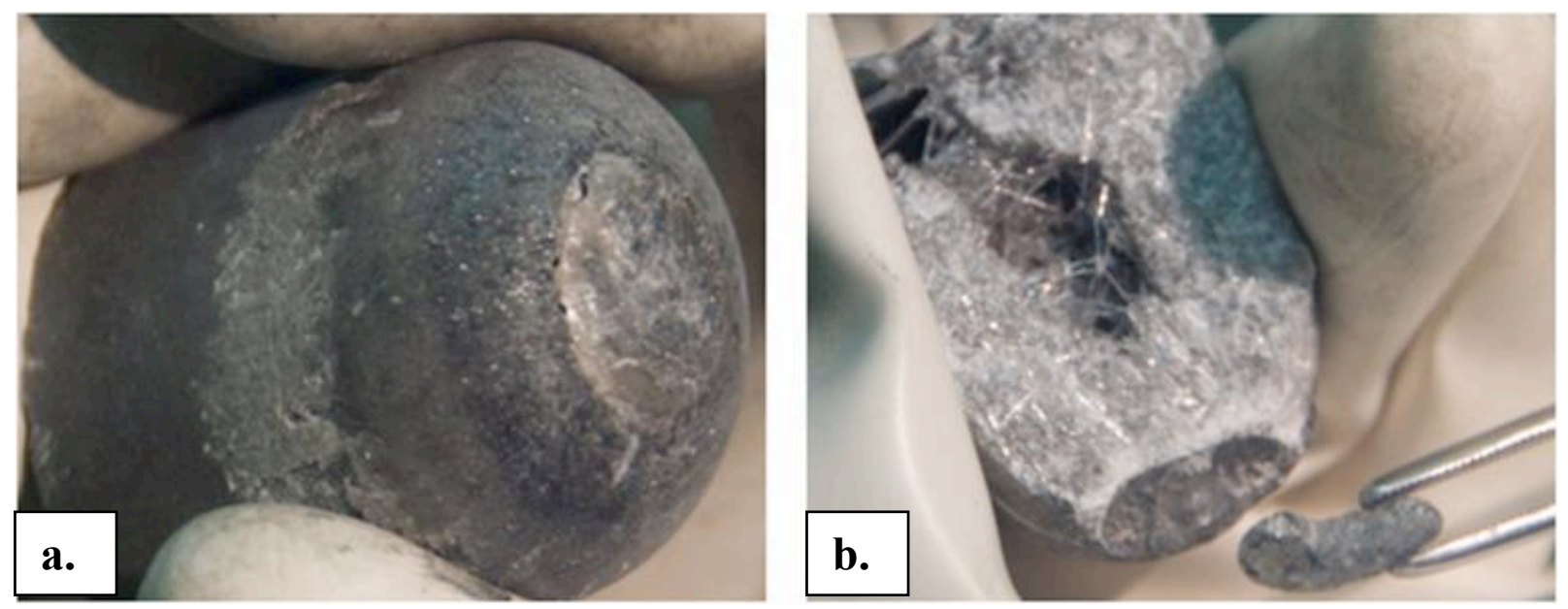

Figure 5.Neptunium metal product (a) The neptunium metal settles to the bottom of the mixture as a readily distinguishable button. (b) The button is easily retrieved from the other materials.

\section{Discussion}

The order of material layers has been tested and found to be extremely important. The first experiments were performed with lithium and lithium chloride, but this system did not achieve the desired reduction. Early experiments with the calcium system involved a two step method whereby the neptunium oxide and calcium metal were heated and then cooled before the calcium chloride was added. 
The optimized order places the least dense material (calcium metal) on the bottom so that when the reagents melt it floats through the denser neptunium oxide layer, thus allowing for maximum mixing (Table 1). The additional contact between the reducing agent (calcium) and the neptunium oxide seems to aid in increasing the yield of the reduction. Density separation also results in the neptunium metal sinking to the bottom of the mixture for easy retrieval.

Also of great importance is the calcium chloride to calcium metal ratio. In order for the reaction to be successful, the ratio must be at least 5 to 1 weight percent, salt to metal. The reaction occurring is as follows:

$$
2 \mathrm{Ca}_{(\text {metal })}+\mathrm{NpO}_{2} \rightarrow 2 \mathrm{CaO}+\mathrm{Np}_{(\text {metal })}
$$

It is thought that as the neptunium oxide is reduced by the calcium metal, the calcium oxide that is being formed creates a passivation layer that will cause the reduction to cease if it is not removed. Literature reports indicate that there is a reduction in the solubility of calcium in calcium chloride as calcium oxide content increases [8]. While the binary $\mathrm{Ca} / \mathrm{CaCl}_{2}$ phase diagram [8] indicates that both are in liquid form at $850^{\circ} \mathrm{C}$, it is important to add excess calcium chloride in order to compensate for the reduction in calcium metal solubility with increased calcium oxide production. In order for the reduction of the neptunium oxide to continue, all of the calcium oxide being formed must be absorbed into the calcium chloride salt, thereby removing it from the reaction zone. If there is not an adequate amount of calcium chloride in the mixture to absorb the calcium oxide as it is formed, the reduction of neptunium oxide will stop. It is also important that the calcium stay dissolved in the calcium chloride salt because if it does not stay in the molten mixture it will float to the top, thus removing it from the reaction zone. The stirring of the mixture is also important to ensure that the calcium has adequate contact with the neptunium oxide. A stoichiometric excess of calcium metal is added to try to overcome the solubility and density obstacles.

The calcium chloride salt used for the reduction must be ultra-dry because any water hinders the reduction process. The original experiments were performed using standard anhydrous calcium chloride salt from Sigma Aldrich and these were unsuccessful. This material was found not to be pure enough for the process. Therefore, ultra-dry, 99.9\% (metals basis) ampoules of calcium chloride from Alfa Aesar are obtained at the start of each reduction run. Due to the sensitivity of the process to moisture and oxygen the ultra-dry calcium chloride is ordered as close as possible to the start of the reduction run and not kept on hand in the laboratory or in the glovebox.

The calcining of the neptunium oxide starting material is also important to remove water and other volatile impurities from the material. It is also necessary to store the reducing metal, calcium in this case, in inert atmosphere in order to prevent it from oxidizing in air prior to contact with the neptunium oxide because, as is the case with the other reagents, the calcium metal needs to be pure. The entire process is performed under inert atmosphere in order to ensure the neptunium metal produced is not re-oxidized. If the reduced metal is exposed to air it will quickly oxidize back to neptunium oxide powder.

The somewhat low yield is thought to be due to metallic material that is dispersed throughout the reaction mixture that did not settle to the bottom. Longer soak times after stirring could produce higher yield. The current set up limits the amount of soak time available as the furnace must be monitored at all times. Glovebox improvements which include the addition of a fire suppression system will allow for longer soak times.

\section{Conclusions}

A relatively high throughput method for reducing neptunium oxide to pure neptunium metal has been developed. Ideally the method would produce a higher yield of metal and would allow for larger batch sizes. Future plans include a redesign of the furnace in order to allow for better monitoring of the reaction and larger batch sizes. It should be noted that depending on the purity of the oxide feedstock available for 
the process the purity of the final product might vary. If the metal produced was not pure enough for fuel casting purposes or if the material were intended for purposes other than fuel fabrication (i.e. characterization for a phase diagram publication) additional purification steps might be necessary. For this reason a step of hydride-dehydride is being explored for purification of the final product.

\section{References}

1. Moser, W.S., James, N.D. (1984). Review of Major Plutonium Pyrochemical Technology. Journal of Less Common Metals, 100, 171-187. doi:10.1016/0022-5088(84)90062-6

2. Perry, G.S., Macdonald, L.G. (1985). Role of $\mathrm{CaCl} 2$ in the Reduction of $\mathrm{PuO} 2$. Journal of Nuclear Materials, 130, 234-241. doi:10.1016/0022-3115(85)90312-5

3. Sakamura, Y., Kurata, M., Inoue, T. (2006). Electrochemical Reduction of UO2 in Molten CaCl2 or LiCl. Journal of The Electrochemical Society, 153, D31-D39. doi: 10.1149/1.2160430

4. Spirlet, J.C., Mueller, W. (1985). Direct Reduction of Actinide Oxide and Carbide to Metal: Application to the Preparation of Plutonium Metal. Nuclear Instruments and Methods in Physics Research, A236, 500-502. doi:10.1016/0168-9002(85)90949-0

5. Choi, E.Y., Won, C.Y., Kang, D.S., Kim, S.W., Cha, J.S., Lee, S.J., Park, W., Im, H.S., Hurr, J.M. (2014). Production of Uranium Metal via Electrolytic Reduction of Uranium Oxide in Molten LiCl and Salt Distillation. Journal of Radioanalytical and Nuclear Chemistry, 304, 535546. doi:10.1007/s10967-014-3842-2

6. Usami, T., Kurata, M., Inoue, T., Simes, H.E., Beetham, S.A., Jenkins, J.A. (2002). Pyrochemical Reduction of Uranium Dioxide and Plutonium Dioxide by Lithium Metal. Journal of Nuclear Materials, 300, 15-26. doi:10.1016/S0022-3115(01)00703-6

7. Usami, T., Kato, T., Kurata, M., Inoue, T., Sims, H.E., Beetham, S.A., Jenkins, J.A. (2002). Lithium Reduction of Americium Dioxide to Generate Americium Metal. Journal of Nuclear Materials, 304, 50-55. doi:10.1016/S0022-3115(02)00853-X

8. Shaw, S., Watson, R. (2009). Solubility of Calcium in CaCl2-CaO. Electrochemical Society Transactions, 16 (49), 301-308. doi: 10.1149/1.3159334

\section{Acknowledgements}

Work funded by the U.S. Department of Energy Office of Nuclear Energy. Special thanks to Dr. James McNeese (LLNL), Scott Wilde (INL), Dr. Jeffrey Giglio (INL), Bryan Forsmann (Boise State University) 